\title{
A Vehicle Path Planning Method
}

\author{
Sun Yong, Li Wen-wei, Liu Tie-zheng and Li Guang-yun \\ China Research and Development Academy of Machinery Equipment; Beijing 100089; China \\ sunyong1984@gmail.com
}

Keywords: path planning, vehicle, method

\begin{abstract}
Based on some assuming and definition, this paper propose a method of calculate a hidden area, and path is planned based on the hidden area and vehicle forward movements type in case of hidden area exist or not, this method is easy and practicality, it can used in people's path planning also.
\end{abstract}

\section{Introduction}

Under fire threat conditions, armored vehicles attacked the choice of route is a path planning problem. In the process of advancing, armored vehicles try to take advantage of topography, surface features, in order to reduce the probability of being found and destroyed. At the same time, but also to facilitate the search and attack enemy targets (for people, mainly hide themselves, to reduce the probability will be found), to solve this problem, complete the following tasks:

\section{Assumptions and definition}

Hidden area defined: our troops can be completely hidden or partially hidden in this area.

Completely hidden: the enemy can't observe our side, and our side can't search for the enemy target and shoot.

Part hidden: our observation equipment and weapon line above the shelter, can searchable aiming and attack enemy targets when reduce the probability of the enemy discovery and destroy.

Whether some point $A$ is the hidden point in this area, is related with the observation point position and height of the enemy, but also with the size of the target at point $A$.

For calculating the hidden area, you need to specify in advance a number (such as 2-3) enemy observation point in the prevention area, if the enemy targets have been detected, places the target actual detection position as the enemy observation point specified location, or predict 2-3 points as the enemy observation point specified location in the area.

Assumed enemy (our) target (vehicle) height is $h *$, and the enemy (our) target observation point height is equal to the height of the fire line, value is $h *$ (also can take different values) (assuming human height is $h *$ ).

Observed degree of enemy $i$-th observation point to the target of point $A$ is defined as the ratio of the point $i$ to point $A$ of the target viewing area (height) and the total area of the target $($ target height $h *)$, referred to as $\rho_{i}\left(0 \leqslant \rho_{i} \leqslant 1, i=1,2,3\right)$.

The hidden degree of point $\mathbf{A}$ is defined as $\rho=1-\frac{1}{3} \sum_{i=1}^{3} \rho_{i}$ or $\rho=\min _{i=1,2,3}\left\{1-\rho_{i}\right\}$

Observed degree of target at point $A$ is defined as the ratio of the point $A$ to point $i$ of the enemy target viewing area (height) and the total area of the target (target height $h^{*}$ ).

A goal at the observation point is defined as the targets viewing area (height) point $\mathrm{A}$ to point $\mathrm{i}$ occupy a total area of target (target height $\mathrm{h} *$ ) ratio, referred to as $\eta \mathrm{i}$, observed degree of target at point $\mathrm{A}$ is defined as $\eta=\frac{1}{3} \sum_{i=1}^{3} \eta_{i}$.

Environmental entities are divided into three categories

Housing, barrier walls, barbed wire, etc. - above the ground, man can't pass. Class I 
Trees, crops, etc. - above the ground, man can't pass, as a hidden area. Class II

The trenches - below ground, man can pass, as a hidden area. ClassIII

According to the position of the entity environment, modify the height values of the corresponding position in the terrain database

\section{Calculate a hidden area}

Set the target observation point coordinates is $\vec{A}=\left(x_{A}, y_{A}, z_{A}+h^{*}\right)$ of point A, the enemy target coordinates is $\left(x_{B}, y_{B}, z_{B}\right)$ of point $\mathrm{B}$, the enemy target height is $\mathrm{h}^{*}$, calculate the observed degree of point A to target at point $B$.

Draw a straight line segment $A B^{\prime}$ ( $A B^{\prime}$ is projection in the horizontal plane of the segment $\left.A B\right)$ :

$$
\begin{aligned}
& y=y_{A}+\lambda\left(x-x_{A}\right), \quad y \in\left(y_{A}, y_{B}\right) \\
& \lambda=\left\{\begin{array}{ccc}
\frac{y_{B}-y_{A}}{x_{B}-x_{A}} & \text { if } \quad x_{B} \neq x_{A} \\
0 & \text { if } \quad x_{B}=x_{A}
\end{array}\right.
\end{aligned}
$$

Set $n$ points $\mathbf{L}_{1}, \ldots, \mathbf{L}_{\mathbf{n}}$ with regular intervals $(\Delta l)$ at line segment $\mathbf{A B} \mathbf{B}^{\prime}, \quad(1+n) \Delta l=\overline{A B}$ For each point $c_{i}$, find its nearest four points in the map database (exactly four vertices of a square, point $c_{i}$ located within this square)

Calculate the height $h_{c i}$ of point $L_{i}$ by the four-point interpolation.

Calculate the slope

$\lambda_{00}=\frac{z_{B}-\left(z_{A}+h^{*}\right)}{(n+1) \Delta l}, \quad \lambda_{01}=\frac{z_{B}-z_{A}}{(n+1) \Delta l}, \quad \lambda_{i}=\frac{h_{c i}-\left(z_{A}+h^{*}\right)}{i \Delta l}, \quad \lambda_{m}=\max _{1 \leq i \leq n}\left\{\lambda_{i}\right\}$

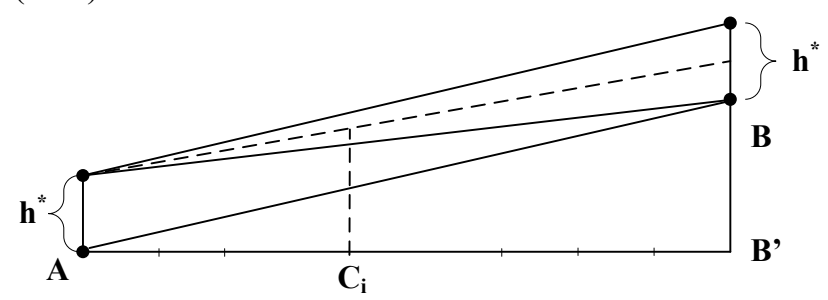

Fig. 1 Calculate the slope.

If $\lambda_{m} \geq \lambda_{01}$, then observed degree is 0 .

If $\lambda_{m} \leq \lambda_{00}$, then observed degree is 1 .

If $\lambda_{00} \leq \lambda_{m} \leq \lambda_{01}$, then observed degree is $\eta=\frac{\operatorname{tg} \lambda_{01}-\operatorname{tg} \lambda_{m}}{\operatorname{tg} \lambda_{01}-\operatorname{tg} \lambda_{00}}$.

Similarly, the observed degree can be calculated of B to A target, hidden degree $\rho(x, y, z)$ and observed degree $\eta(x, y, z)$ of any point $(x, y, z)$ on the battlefield can be calculated.

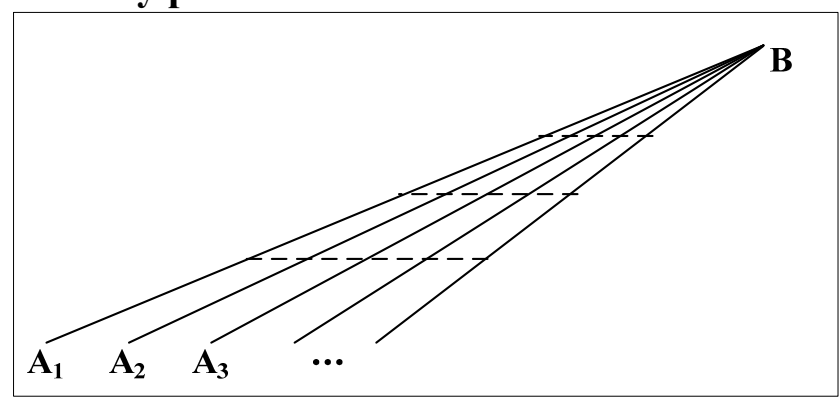

Fig. 2 Calculate hidden degree and observed degree. 
Draw ray from point $B$ to the possible vehicle attack starting point $A_{1} 、 A_{2} \ldots$, and calculate hidden degree of the points on the rays $B A_{i}$ to point $B$.

Determine the hidden area

Definition: The point $A$ is the hidden point, if $\rho(A) \geq \rho_{0},\left(\rho_{0}\right.$ is $0.7,0.8$, etc. $)$.

Definition: A connectable area is hidden area, if all points within the area are the hidden point.

For people

Class II, III entities area (trees, crops, trenches, etc.) is a hidden area.

Point A of battle zone is class I hidden point, if $\rho(A) \geqslant 0.7$ (ie people stoop hidden).

Point A of battle zone is class II hidden point, if $\rho(A) \geqslant 0.3$ (ie people crawl hidden).

Set of all the connectable hidden point is hidden area, apparently a class I hidden area is a subset of class II hidden area.

\section{Tank unit forward movements under enemy fire threat}

Promoting: unit non-stop attacking advances, suitable for flat terrain, open, to convenient vehicle movement not hide.

Bounding: unit segment successive attack advances, suitable for enemy fire is strong, we can use terrain of the impact direction.

Alternate cover bounding: each unit alternately cover, successive bounding, suitable for strong enemy fire, our superiors fire cannot reliably suppress enemy and narrow front of attacking, complex terrain.

The way of attack forward is decided by battlefield hidden area, if no hidden areas on the attack line, only choose promoting, if multiple hidden areas on the attack line, choose bounding or alternately cover bounding.

For people, the action include normal movement forward, bending forward, high position creeping, low position creeping, running forward.

\section{Path planning in case of hidden area}

In two steps, the first step, the start and end points, each hidden areas are first-degree node respectively, multiple entry point of larger hidden areas edge are selected as first-degree node. The movement price of nodes can be ignored in the same hidden area. If the hidden area is long (such as trench), we can set two (at both ends of the trench) or three (at both ends and middle) nodes. If length and width is similar, we can set $4-6$ nodes at the area edge. Recorded the first-degree node as a node $A_{1}, \ldots, A_{k}$, for node $A_{i}$, calculates the optimal path node to its neighboring nodes $A_{j}$, since the hidden area is constituted by multiple points, the optimal path of nodes representing two hidden area is defined is the optimum path between two hidden area; the second step, first-degree node $A_{1}, \ldots, A_{k}$ constitute road network, the optimal path between the beginning and the end are calculated according to the road network.

Seeking the neighbor nodes of first-degree node $A_{i}(i=1, \ldots, k)$.

The node $A_{i}$ is used as the origin (If a hidden area include multiple points, the node $A_{i}$ is representative of a hidden area, then $\mathrm{A}_{\mathrm{i}}$ is the center point)and make 8 rays, angles of rays and $\mathrm{X}$-axis are $0^{\circ}, 45^{\circ}, 90^{\circ}, 135^{\circ}, 180^{\circ}, 225^{\circ}, 270^{\circ}, 315^{\circ}, 8$ rays constituting eight, find the nearest first-degree node from the origin $\mathrm{Ai}$ in each area, then $\mathrm{A}_{\mathrm{ij}}(\mathrm{j}=1, \ldots, 8)$ constituting the neighborhood set of node $\mathrm{A}_{\mathrm{i}}$. If minimum distance from a hidden area in fan-shaped areas to $A_{i}$ exceeds threshold $D_{0}$, then give up; if the distance between two hidden areas less than the threshold $\mathrm{D}_{01}$, then two hidden areas are considered connectable. 


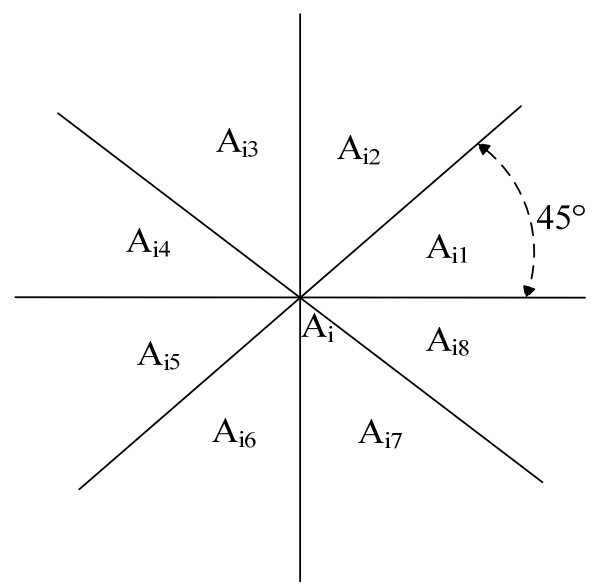

Fig. 3 Seeking the neighbor nodes.

\section{Seeking optimal path from first-degree node $A_{i}$ to the neighborhood node $A_{i j}(j=1, \ldots, 8)$}

\section{Determine the grid area}

Seeking external rectangle of 9 hidden area,

Order uppermost point of area $A_{i 1}, A_{i 2}, A_{i 3}, A_{i 4}$ is $y_{M}$

Order lowermost point of area $A_{i 5}, A_{i 6}, A_{i 7}, A_{i 8}$ is $y_{m}$

Order leftmost point of area $A_{i 3}, A_{i 4}, A_{i 5}, A_{i 6}$ is $x_{m}$

Order rightmost point of area $A_{i 1}, A_{i 2}, A_{i 7}, A_{i 8}$ is $x_{M}$

The 4 straight lines $\mathrm{x}=\mathrm{x}_{\mathrm{m}}, \mathrm{x}=\mathrm{x}_{\mathrm{M}}, \mathrm{y}=\mathrm{y}_{\mathrm{m}}, \mathrm{y}=\mathrm{y}_{\mathrm{M}}$ intersect and form 9 external rectangle of hidden area, the road network is constructed based on the grid point in the rectangle.

\section{Analyzing connectivity}

From left to right, from bottom to top, the following work is implemented for each grid point: determine the connectivity between the points and adjacent grid point (total of eight), if not connectable, cancel it from the adjacent points, if connectable, calculate the road travel cost, and build the road network.

\section{Constructing the road network}

Set first-degree node $\mathrm{Ai}$ as the starting point, hidden area $\mathrm{A}_{\mathrm{ij}}(\mathrm{j}=1, \ldots, 8)$ as the end point, optimal path is calculated with $\mathrm{D}$ algorithms from $\mathrm{A}_{\mathrm{i}}$ to each adjacent hidden area (not ever find and connectable hidden area), the optimal path of a point to an area is obtained as follows: when the first encounter the grid point of the area with $\mathrm{D}$ algorithms calculation (if $\mathrm{D}$ algorithm finding price exceeds the threshold value $\mathrm{D}_{0}$ yet not find a hidden area in that direction, then give up), then the optimal path of $A_{i}$ to the hidden area is known, it is the optimal path of $A_{i}$ to that grid points.

\section{Seeking an optimal path between any two nodes}

\section{Constructing the road network}

The road network between each hidden area constructed has been completed;

For a given battle zone starting point $A_{0}$ and end point $A_{01}$, optimal path of point $A_{0}\left(A_{01}\right)$ to the adjacent hidden area are calculated.

Road network are constructed by nodes $\mathrm{A}_{0}, \mathrm{~A}_{01}$ and each hidden area.

The optimal path of $A_{0}$ to $A_{01}$ is calculated with the $A^{*}$ algorithm, wherein the path between two points is equivalent to the formula

$$
Q=W_{1} \cdot P_{1}(T, \bar{R}) \cdot C_{1}-W_{2} \cdot P_{2}(T, \bar{R}) \cdot C_{2}
$$

Where $\mathrm{T}$ is the path motion time between two points, $\bar{R}$ is the path case between two points, $\mathrm{P}_{1}$ is our vehicle destroyed probability in the time $\mathrm{T}, \mathrm{P}_{2}$ is enemy target destroyed probability in the time $\mathrm{T}$, $\mathrm{C}_{2}$ and $\mathrm{C}_{1}$ are enemy's and our target value, $\mathrm{W}_{1}$ and $\mathrm{W}_{2}$ are the corresponding weights, the expense $\mathrm{Q}$ is smaller the better.

For people, the impact of the movement against the enemy is not considered, then $Q=W_{1} \cdot P_{1}(T, \bar{R}) \cdot C_{1}$. 


$$
P_{1}(T, \bar{R})=\left\{\begin{array}{cc}
0 & T \leq T_{0} \\
1-e^{-\lambda\left(T-T_{0}\right)} & T>T_{0}
\end{array}\right.
$$

Where the parameter $\lambda$ is related to factors of enemy fire control precision, fire intensity, the average hidden degree of our vehicle passed point, protection capability, enemy shooting validity (i.e. the angle of enemy with me connection and my speed direction) and the average distance from enemy fire and other factors, in the case of two sides of the weapons identified, $\lambda$ is a function of the average hidden degree and average distance. T0 is the reaction time of the enemy weapon (including search discovery time, the fire control system launch time and projectile flight time).

$$
P_{2}(T, \bar{R})=\left\{\begin{array}{cc}
0 & T \leq T_{01} \\
1-e^{-\lambda_{01}\left(T-T_{01}\right)} & T>T_{01}
\end{array}\right.
$$

Where the parameter $\lambda_{01}$ is related to factors of our fire control precision, fire intensity, averaged observed degree of passed point, the enemy vehicle protection capability, both distance and other factors. $\mathrm{T}_{01}$ is our weapons reaction time.

\section{Path planning in case of no hidden area}

\section{Determine the grid area}

Based on the forecast of enemy weapons type and arming situation, our starting position and attack area range are determined, and the grid area is determined.

\section{Constructing the road network}

Similar to "Analyzing connectivity".

\section{Optimal Path}

Set vehicle assault starting point as a starting point, a point of $500 \mathrm{~m}$ from enemy fire forefront as the end point, optimal path is calculated with the $\mathrm{A}^{*}$ algorithm, path cost between two grid points is the vehicle travel time

\section{References}

[1] Li Wen-wei, Meng Hong, Sun Yong, "Simulation of Optical Imaging Reconnaissance to Ground," 2012 National Conference on Information Technology and Computer Science (CITCS2012), 2012.

[2] SUN Li-jun HU Xiang-pei WANG Zheng, “Reviews on Vehicle Routing Problem and Its Solution Methods,” SYSTEMS ENGINEERING: 2006, 24(11).

[3] YAN Ming LI Wen-qing ZHOU Tao, “All Path Programming for Tank CGF Based on A*Arithmetic,” COMMAND CONTROL \& SIMULATION: 2008, 30(3).

[4] Han Zhi-jun, Hua Chuan-jie, Wang Lei, “Path Planning for CGF Entities' Intelligent Behavior Using A* Algorithm,” COMPUTER ENGINEERING AND APPLICATIONS: 2003, 39(35).

[5] SUN Shao-bin, WANG Kuan-quan, LIN Xue-hua, “Algorithm for CGF Real-time Route Replanning in Dynamic Environment,” JOURNAL OF SYSTEM SIMULATION: 2007, 19(13).

[6] YAN Shou-zhu, XUE Qing, LUO Jia, "Research of Wheeled Armored Vehicle CGF Path Planning Based on Immune Genetic Algorithm,” Journal of Sichuan Ordnance: 2014, 35(10). 